\title{
Ein Markt mit Milliarden-Potenzial
}

\author{
Was das Reich der Mitte dringend braucht, sind umweltgerechte
}

Technologien - auch in der industriellen Teilereinigung.

U nter dem enormen Wirtschaftswachstum der vergangenen Jahre in China hat die Umwelt besonders stark gelitten. Dies hat zu einer Reihe neuer Gesetze und Vorschriften geführt, die den Umweltschutz verbessern und den nachhaltigen Umgang mit Ressourcen fördern. Sie beinhalten unter anderem, dass Unternehmen, die Schadstoffe in Gewässer oder die Luft ableiten, dafür eine Genehmigung vorweisen müssen, an die Kontrollanforderungen gekoppelt sind. Außerdem wurde Ende 2011 „The Industry Cleaning Association of China“ (ICAC) gegründet, in der alle Bereiche industrieller Reinigung zusammengefasst sind. Diese Vereinigung wird als treibende Kraft für die Weiterentwicklung von Reinigungstechnologien in China gesehen - auch in der industriellen Teilereinigung.

\section{Unterschiedliche Technologien im} Einsatz

Verschiedene deutsche Hersteller von Reinigungsanlagen importieren bereits seit Jahren Systeme ins Reich der Mitte - Abnehmer sind meist die chinesischen Niederlassungen westlicher Konzerne. Diese Anlagen entsprechen üblicherweise den Umwelt-Standards, die auf den heimischen Märkten der Anlagenbauer beziehungsweise -betreiber gefordert werden.

Auf dem chinesischen Markt hat sich der Bedarf an industrieller Reinigungstechnologie in den letzten Jahren enorm erhöht. Im Einsatz sind dabei einerseits noch klassische Verfahren, die manuell oder in halbautomatischen Anlagen durchgeführt werden. Andere Technologien wie beispielsweise umweltverträgliche nasschemische Reinigungsverfahren, Wasserstrahl- und $\mathrm{CO}_{2}$-Reinigung, Laserstrahlreinigung sowie der Einsatz biologischer Reiniger entwickeln sich nach Informationen der ICAC allmählich.

Andererseits gibt es Branchen wie die Automobilindustrie, die bei der Teilereinigung den Trends europäischer Hersteller folgt und vergleichbare Anforderungen hinsichtlich der zu erreichenden Sauberkeit sowie der Umweltverträglichkeit der eingesetzten Reinigungsverfahren stellt.

\section{Steigender Bedarf in zahlreichen Branchen}

Durch das auch für die kommenden Jahre angestrebte Wirtschaftswachstum von mindestens 7,5 Prozent jährlich und der Anstrengungen, Arbeitsund Umweltschutz zu verbessern, steigt der Bedarf an moderner industrieller Reinigungstechnologie in China. Als Branchen, die davon betroffen sind, sieht die ICAC unter anderen die petrochemische und chemische Industrie, die Metallverarbeitung, den Maschinenbau, das Verkehrswesen, die Elektro- und elektronische Industrie, die Energieerzeugung sowie die Druck- und Textilindustrie. Das chinesische Ministerium für Industrie und Informationstechnologie beziffert das Marktvolumen für industrielle Reinigungstechnik auf über 20 Billionen Yuan (rund 2,5 Milliarden Euro) jährlich.

We sentli che Kriterien bei der Optimierung der Reinigungsprozesse stellen die Verwendung geschlos- sener Kreislaufanlagen, ein geringer Energie- und Wasserverbrauch, Reduzierung von Emissionen, Verbesserungen im Bereich der Sicherheit, Produktionsgeschwindigkeit, Produktqualität und Lebensdauer dar.

\section{7-Jahres-Plan zur Entwicklung innovativer Lösungen}

Vor kurzem hat die ICAC einen Leitfaden zur Entwicklungsplanung in der industriellen Reinigungstechnologie für die kommenden sieben Jahre veröffentlicht. Danach sollen die Pulse-Technologie, die Ultraschallreinigung und das pneumatische Reinigungsstrahlen bis 2020 große Schritte nach vorne machen. Die Richtung dabei heißt High-End und Grün. Entsprechend sollen hoch leistungsfähige und umweltgerechte Reinigungstechnologien und -produkte mit hohem Mehrwert für unterschiedlichste Bereiche entwickelt werden, mit denen die Industrialisierung vorangetrieben werden kann.

Mit ihren Anlagen und Produkten für die industrielle Teilereinigung können aber sicher auch deutsche Hersteller auf dem chinesischen Markt punkten.

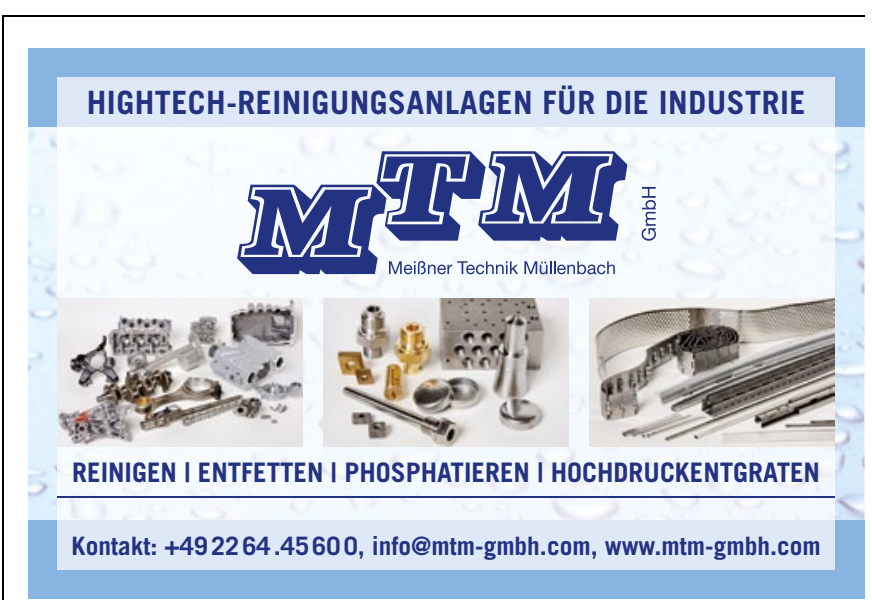

